\title{
THE DISTANCE TO THE GALACTIC CENTER: $R_{o}$
}

\author{
MARK J. REID \\ Harvard-Smithsonian Center for Astrophysics \\ 60 Garden Street \\ Cambridge, Massachusetts 02138
}

\begin{abstract}
Great progress has been made toward measuring the size of the Milky Way. There are now several methods that employ independent calibrations to estimate the distance to the center of the Galaxy, $R_{o}$, and these methods have been applied to many types of astronomical objects. $R_{o}$ estimates generally have been decreasing over the last 15 years. At this time a reasonable "best value" estimate for $R_{o}$ is $7.7 \pm 0.7 \mathrm{kpc}$.
\end{abstract}

\section{Introduction}

In 1918 Harlow Shapley published a landmark paper on the distribution of globular clusters in the Milky Way. He found that globular clusters were most concentrated at a distance of $\approx 13 \mathrm{kpc}$ toward Sagittarius at $\ell^{I}=325^{\circ}$. Shapley correctly suggested that the Galactic Center resided near the center of this distribution and not at the apparent maximum of the stellar distribution (which later was understood to be strongly affected by absorption). This work was the first major step leading to the currently adopted value for $R_{o}$, the distance to the Galactic Center, of $8.5 \mathrm{kpc}$ (Kerr and Lynden-Bell 1986).

Why do we care about $R_{o}$ ? A change in the value of $R_{o}$ has widespread impact on astrophysics. The following is a brief list of items that would be affected by changing $R_{o}$ :

1) all kinematic distances;

2) the mass of the Galaxy and the Galactic Center;

3) the luminosity of most stars and some X-ray sources that appear superEddington for large $R_{o}$ values;

4) extragalactic distances by

a) recalibrating the absolute magnitudes of $R R$ Lyrae variables, globular clusters, Cepheids, OB stars and Mira variables, and

b) moving spiral galaxies closer to or further from the Sun to match Milky Way size (e.g., de Vaucouleurs 1983); 
5) the "dark matter" in the Local Group by affecting the Andromeda infall speed if $\Theta_{o}$ is also adjusted (Trimble 1986).

In Section 2 of this review we discuss estimates of $R_{o}$ from papers published since 1974. This section includes a new method of directly measuring $R_{o}$ from $\mathrm{H}_{2} \mathrm{O}$ maser proper motions. In Section 3 we combine estimates of $R_{o}$ to give a "best value," taking into account statistical as well as systematic uncertainties. Finally, in Section 4 we mention some examples of observations now being conducted, or possible in the near future, that will greatly improve our estimate of $R_{o}$.

\section{Review of the Determinations of $R_{o}$}

\subsection{DIRECT MEASUREMENT}

We define a direct measurement of $R_{o}$ as a distance measured without a "standard candle" calibration or galactic rotation model to a source at or very near the Galactic Center. Presently this has been done only for the $\mathrm{H}_{2} \mathrm{O}$ maser source in Sgr B2(N).

\subsection{1 $\mathrm{H}_{2} \mathrm{O}$ proper motions in $\mathrm{Sgr} \mathrm{B2}$}

Interstellar masers occur in the envelopes of newly formed massive stars. $\mathrm{H}_{2} \mathrm{O}$ molecules are a trace constituent of these envelopes, and population inversion of the molecular energy levels followed by coherent de-excitation causes the appearance of masing "spots" of emission $\sim 10^{13} \mathrm{~cm}$ in size with brightness temperatures as high as $10^{15} \mathrm{~K}$. Because of the small sizes and high brightnesses of interstellar maser spots, they are amenable to precise astrometric measurements that allow their proper motions to be determined. VLBI techniques have achieved a relative positional accuracy of $\sim 10$ micro-arcsec ( $\mu a s)$ across fields of size $\sim 3$ arcsec. This is sufficient to determine proper motions and estimate distances throughout the Galaxy.

Proper motion studies of the Sgr B2(N) water masers in the galactic center region (Reid et al. 1988) indicate that the $\mathrm{H}_{2} \mathrm{O}$ maser spots are expanding, presumably in an energetic stellar wind from a newly formed O-type star. The maser spots are observed to move along straight lines on the sky. The three measured motions (two dimensions of proper motion and the radial motion from Doppler shifts) are modelled as a uniformly expanding spherical source, and a least-squares fit to the data yields an estimate of the distance to the source of $7.1 \pm 1.5 \mathrm{kpc}$. Sgr B2(N) is almost certainly within $0.3 \mathrm{kpc}$ of the Galactic Center (see discussion in Reid et al. 1988) and, thus, the Sgr B2(N) distance can be directly used as an estimate of $R_{o}$. 


\subsection{SECONDARY MEASUREMENTS}

Secondary measurements use "standard candle" distances to objects whose distributions are assumed to be symmetrical about the Galactic Center.

\subsubsection{Globular Clusters}

Table 1. Globular Clusters

$\begin{array}{llll}\text { REFERENCE } & R_{\circ}(\mathrm{kpc}) & \text { CALIBRATION } & \text { COMMENTS } \\ \text { Harris 1976,1980 } & 8.5 \pm 1.6 & \mathrm{M}_{V}(\mathrm{HB})=0.6 & \text { using means, } \mathrm{Z}_{\text {lim }} \\ \text { de Vaucouleurs \& Buta 1978 } & 7.0 & \mathrm{M}_{V}(\mathrm{RR})=0.86 & \text { Harris's method } \\ \text { Frenk \& White 1982 } & 6.2 \pm 0.9 & \mathrm{M}_{V}(\mathrm{HB})=0.6 & \text { low metallicity } \\ & 9.1 \pm 1.4 & \mathrm{M}_{V}(\mathrm{HB})=0.6 & \text { high metallicity } \\ & 7.2 \pm 1.1 & \mathrm{M}_{V}(\mathrm{HB})=1.1 & \text { n } \\ \text { Sasaki \& Ishizawa 1978 } & 9.2 \pm 1.3 & \mathrm{M}_{V}(\mathrm{RR})=0.6 & \text { cone of avoidance } \\ \text { Surdin 1980 } & 10.1 \pm 0.7 & \mathrm{M}_{V}(\mathrm{RR})=0.6 & \text { metallicity distrib. }\end{array}$

2.2.1.1 CENTROID OF DISTRIBUTION: This technique assumes that globular clusters are symmetrically distributed about the Galactic Center. Therefore, if one plots the number of clusters versus distance from the Sun (toward the Galactic Center), the peak should occur near the distance of the Galactic Center. Table 1 lists some recent estimates of $R_{o}$ from globular clusters. There is considerable controversy over the question of the absolute magnitude of the horizontal branch, $M_{V}(\mathrm{HB})$, as a function of cluster metallicity. $\mathrm{M}_{V}(\mathrm{HB})$ differences of up to $0.5 \mathrm{mag}$, or a factor of about 25\%, are involved. Also extinction is a problem at low galactic $Z$ 's (i.e., distance from the galactic plane) and for distant clusters at moderate $Z$ 's. Finally, there is disagreement on biases introduced in the statistical procedures used to estimate $R_{o}$ from the distributions. Note, for example, that the estimates of $R_{o}$ by Harris $(1976,1980)$ and Frenk and White (1982) are based on essentially the same data set.

2.2.1.2 CONE OF AVOIDANCE: Wright and Innanen (1972) noted that the density of globular clusters diminishes in a cone with a $\sim 15^{\circ}$ opening angle whose axis is aligned with the galactic rotation axis. Sasaki and Ishizawa (1978) suggest that tidal interactions with the galactic center region will preferentially disrupt clusters located along the galactic rotation axis. They claim that $R_{o}=9.2 \pm 1.3 \mathrm{kpc}$ maximizes the cone angle and that this procedure indicates the distance of the Galactic Center. This distance seems to be based upon $M_{V}(R R)=0.6$ with no note of the metallicity of clusters used.

2.2.1.3 METALLICITY DISTRIBUTION: The metallicity of globular clusters decreases with distance, $R$, from the Galactic Center. Surdin (1980) points out that if the 
globular cluster distribution is axially symmetric about the galactic rotation axis, then $R_{o}$ can be estimated by adjusting its value until the cluster metallicity is uncorrelated with galactocentric azimuth. He points out that metallicity estimates are not strongly affected by extinction corrections, thus avoiding this source of systematic error. Surdin estimates a value of $R_{o}=10.1 \pm 0.7 \mathrm{kpc}$, averaging catalog distances based on $\mathrm{M}_{V}(\mathrm{RR})=0.6$ for all metallicities and a metallicity dependent $M_{V}$. The quoted statistical error seems to be considerably underestimated, since it is estimated from the scatter in a plot of the correlation coefficient (of metallicity with galactocentric azimuth) versus $R_{o}$. Because the same data set is used for each point in that plot, the points are correlated, leading to an underestimate of the variation in $R_{o}$ that would arise were an ensemble of globular cluster data sets available.

\subsubsection{RR Lyrae Variables}

Individual RR Lyrae variables can be seen across the Galaxy and toward the Galactic Center through fortuitous "windows" of low extinction such as Baade's Window. Thus, $R_{o}$ can be estimated by finding the distance toward the Galactic Center of the peak of the distribution of RR Lyrae variables (similar to the method used for globular clusters). Table 2 summarizes recent RR Lyrae results.

Table 2. RR Lyrae Variables

$\begin{array}{llll}\text { REFERENCE } & R_{o}(\mathrm{kpc}) & \text { CALIBRATION } & \text { COMMENTS } \\ \text { Oort \& Plaut } 1975 & 8.7 \pm 0.6 & \mathrm{M}_{p g}=0.7 & \\ \text { Clube \& Dawe 1980 } & 7.0 \pm 1.0 & \mathrm{M}_{V}(\mathrm{RR})=1.0 & \\ \text { Blanco \& Blanco } 1985 & 8.0 \pm 0.7 & \mathrm{M}_{V}(\mathrm{RR})=0.6 & \text { all metallicities } \\ & 6.9 \pm 0.6 & \mathrm{M}_{V}(\mathrm{RR})=\mathrm{f}\left(\left[\frac{\mathrm{F}_{f}}{\mathrm{H}}\right]\right) & \\ \text { Walker \& Mack 1986 } & 8.1 \pm 0.4 & \mathrm{M}_{V}(\mathrm{RR})=0.6 & \end{array}$

In large part $\mathrm{M}_{V}(\mathrm{HB})$ is tied to $\mathrm{M}_{V}(\mathrm{RR})$ and hence globular cluster distances are correlated with those of RR Lyrae variables. As for globular clusters, $M_{V}(\mathrm{RR})$ as a function of metallicity is not well known. Also, extinction is significant and variable across these windows. Finally, crowding of stellar images in these very densely populated fields can lead to inaccurate measures of apparent magnitude.

\subsubsection{Red Giants and Miras}

Bright stars, other than RR Lyrae variables, also can be seen through interstellar windows. For example, Mira variables (Glass and Feast 1982) are a particularly attractive class of stars for estimating $R_{o}$, since they are luminous and can be observed with moderate sized telescopes. Also, they are bright at infrared wavelengths where the effects of extinction are greatly reduced. Table 3 gives recent estimates of $R_{o}$ from red giant stars. 
Table 3. Red Giants

\section{REFERENCE}

van den Bergh \& Herbst 1974

Glass \& Feast 1982

$R_{o}(\mathrm{kpc})$
$9.2 \pm 2.2$
8.8
7.9

CALIBRATION COMMENTS

$\mathrm{M}_{V}(\mathrm{RR})=0.5 \quad$ Red Giants

$\mathrm{M}_{\text {bol }}(\mathrm{P}=0)=0.76 \quad$ Miras; LMC calib

$\mathrm{M}_{\text {bol }}(\mathrm{P}=0)=0.54$ gal. calib

The calibration of the red giant data of van den Bergh and Herbst (1974) is tied to RR Lyrae variables, and hence does not give a truly independent estimate of $R_{o}$ from that of $R R$ Lyrae variables or globular clusters. The zero-point, $M_{b o l}(P=0)$, in the period-luminosity calibration of the Miras differs by 0.22 mag depending on whether one adopts a galactic calibration or one based on the Large Magellanic Cloud distance (determined from Cepheid variables).

\subsection{INDIRECT MEASUREMENTS}

Indirect determinations of $R_{o}$ combine observations with either a model of the Galaxy or some other theoretical constraints. For example, one approach is to assume a fixed (e.g., Eddington) luminosity and determine distances from observed fluxes for a class of objects.

\subsubsection{Rotation Models of the Galaxy: using $A$ or $\Theta_{0}$}

Table 4. Cepheids, OB stars, HII regions, etc.

$\begin{array}{ll}\text { REFERENCE } & R_{o}(\mathrm{kpc}) \\ \text { Cruz-Gonzalez 1974 } & 8.9 \pm 0.5 \\ \text { Bologna \& Feast 1974 } & 9.0 \\ \text { Crampton et al. 1976 } & 8 \\ \text { Byl \& Ovenden 1978 } & 10.4 \pm 0.5 \\ \text { Caldwell \& Coulson 1987 } & 7.8 \pm 0.7 \\ \text { Quiroga 1980 } & 8.4 \\ \text { Brand 1986 } & 8.0 \pm 0.5 \\ \text { Rohlfs et al. } 1986 & 7.9 \pm 0.7 \\ \text { Herman et al. 1985 } & 8.1 \pm 1.1 \\ \text { Backer \& Sramek 1986 } & 8.5 \pm 1.0 \\ \text { Caldwell \& Ostriker 1981 } & 8.2 \\ \text { Toomre 1972; Rybicki } \text { et al. } 1974 & 8\end{array}$

\begin{tabular}{|c|c|}
\hline ASSUMPTIONS & COMMENTS \\
\hline$A=15 \mathrm{~km} / \mathrm{s} / \mathrm{kpc}$ & stars $<25$ pc distant \\
\hline \multicolumn{2}{|c|}{$A=16.8 \mathrm{~km} / \mathrm{s} / \mathrm{kpc}$ OB stars } \\
\hline \multirow[t]{4}{*}{$A=16.8 \mathrm{~km} / \mathrm{s} / \mathrm{kpc}$} & OB stars/solar circle \\
\hline & mostly OB stars \\
\hline & Cepheids \\
\hline & HI vs OB stars \\
\hline \multirow[t]{2}{*}{$\Theta_{o}=220 \mathrm{~km} / \mathrm{s}$} & HII regions \\
\hline & HII regions \\
\hline$\Theta_{0}=220 \mathrm{~km} / \mathrm{s}$ & OH/IR stars \\
\hline \multirow[t]{2}{*}{$\Theta_{o}=220 \mathrm{~km} / \mathrm{s}$} & Sgr A*motion \\
\hline & Modelling \\
\hline$A-B=25 \mathrm{~km} / \mathrm{s} / \mathrm{kp}$ & pc $\quad n$ \\
\hline
\end{tabular}

Stars and atomic and ionized clouds that partake in the galactic rotation and have estimated distances can, in the context of a model of the Galaxy, be used to estimate $R_{o}$. For example, radial velocity measurements for a sample of stars can be used with a kinematic model for the Galaxy to derive kinematic distances. These distances can be compared with luminosity distances and brought in to agreement 
by adjusting $R_{o}$ (since kinematic distances scale directly with $R_{o}$ ). There are a great variety of objects and analysis techniques in the literature. Table 4 summarizes some of the recent results; consult the original papers for discussions of the distance calibrations for the various objects. (Note the result of Herman et al. (1985) has been rescaled for $\Theta_{o}=220 \mathrm{~km} / \mathrm{s}$.)

Thackeray (1972) and Crampton et al. (1976) point out that there is a sizeable difference in the kinematic properties and, hence, $R_{o}$ values inferred from stars in the northern and southern portions of the Galaxy. Northern stars tend to yield $R_{o}$ values about 3 to $4 \mathrm{kpc}$ smaller than southern stars. Byl and Ovenden (1978) claim to reconcile some of this difference by accounting for noncircular motions associated with spiral structures. It is important to remember that most of these methods are sensitive to local deviations from noncircular motions and/or to sizeable extinction corrections.

\subsubsection{Eddington Luminosity Limit}

Ebisuzaki et al. (1984) estimate the luminosity of a sample of X-ray bursters. Assuming that the emission is associated with a $1.4 M_{\odot}$ compact object (e.g., a contact binary containing a neutron star) and that the emission is at the Eddington limit, they derive "luminosity distances" for the sample. The distribution for 27 bursters peaks toward the Galactic Center at a distance of $6 \pm 2 \mathrm{kpc}$. This can be taken as an estimate of (or an upper limit to) $R_{o}$, provided the emission is at (or below) the Eddington limit.

Cyg X3 is a contact binary containing one and possibly two compact objects. It is a strong, periodic X-ray and radio source. $\mathrm{HI}(21 \mathrm{~cm}$ wavelength) studies (Dickey 1983) indicate that all galactic $\mathrm{HI}$ emission lines are seen in absorption against its continuum radio emission, implying a kinematic distance of at least $1.16 R_{o}$. If the (X-ray) emission from Cyg X3 is sub-Eddington from a 1.4 $M_{\odot}$ object, then Molnar (1985) finds a "luminosity distance" limit of $9.0 \mathrm{kpc}$, suggesting $R_{o} \leqslant 7.7 \mathrm{kpc}$. (We adopt this value as a distance estimate, even though strictly speaking it is an upper limit.)

The critical assumptions used to estimate (or limit) $R_{o}$ from the X-ray bursters and Cyg X3 are that the emissions are at (or below) the Eddington limit and that the compact objects ultimately responsible for the emissions have typical neutron star masses of $1.4 M_{\odot}$. While the former assumption seems reasonable, Molnar et al. (1988) give evidence that, at least for Cyg X3, a more massive object (probably a black hole) is involved.

\section{A "Best Value" for $R_{o}$}

It is not possible to combine all existing estimates of $R_{o}$ to form a "best value" in a statistically rigorous manner. This would require knowledge of the variancecovariance matrix for the set of $R_{o}$ estimates. Unfortunately, we do not have 
reliable values of the uncertainty for each estimate because systematic sources of error are poorly known and often not even discussed. In addition to not knowing the variances, we have only a qualitative understanding of the covariances among the different $R_{o}$ estimates. For example, a change in the RR Lyrae absolute magnitudes directly affects the calibration of absolute magnitudes for globular clusters and to some extent for other stars such as red giants and Cepheids. Thus, the covariances among different methods of determining $R_{o}$ are substantial.

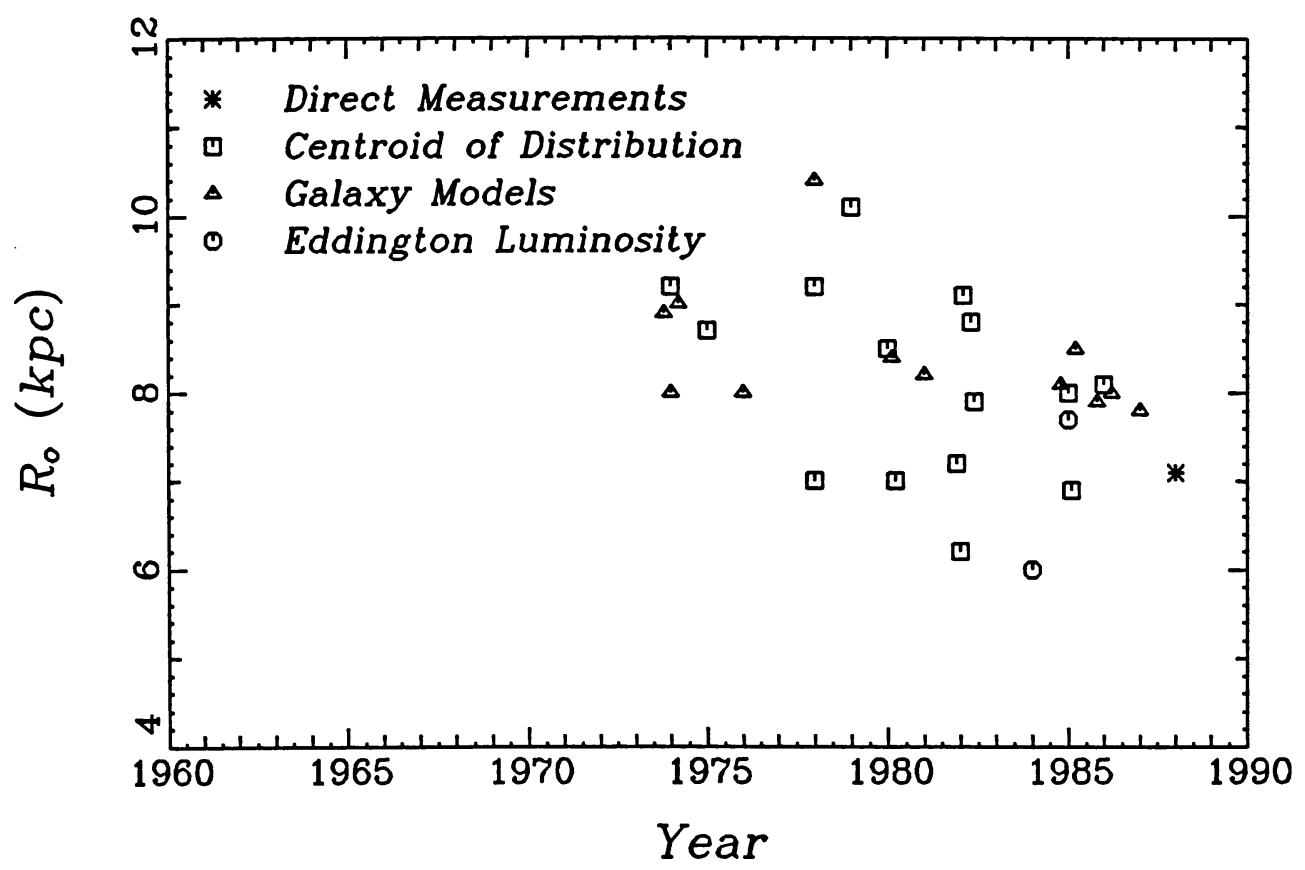

Figure 1. Estimates of the distance to the Galactic Center versus publication date since 1974.

In Figure 1, we plot $R_{0}$ versus publication date from the results cited in this review. Based upon this plot, a case could be made for a statistically significant decrease in estimates of $R_{o}$ with time. In part, this effect is a result of changes in the complex and interrelated distance calibrations for different stars. However, one could also speculate that a significant "bandwagon effect" is operative here. Statistical analyses of (usually incomplete) astronomical data are not straightforward, and current wisdom as to the "correct answer" can affect estimates of $R_{o}$. Faced with these problems Kerr and Lynden-Bell (1986) adopted the simplest approach to finding a "best value" for $R_{o}$ and calculated an unweighted average of recent $R_{o}$ values. We will adopt a different approach, trying to account, in an admittedly crude manner, for statistical and systematic errors, as well as for 
the covariances among different methods.

Table 5 groups $R_{o}$ values based upon four methods that have nearly independent calibrations. Each of these groups are further subgrouped by the stars or sources used to estimate $R_{o}$. Each entry in the table contains an unweighted mean value of $R_{o}$ for that star or source category (from data given in Tables 1 to 4) and a statistical uncertainty that approximately reflects the precision of the technique. The variance-weighted average of $R_{o}$ for each of the four groups is indicated along with its formal uncertainty.

Table 5. $R_{o}$ by Methods

\begin{tabular}{|c|c|c|c|}
\hline METHOD & $\begin{array}{l}R_{o} \\
\text { (kpc) }\end{array}$ & & \\
\hline \multicolumn{4}{|l|}{ Direct Measurement: } \\
\hline \multirow[t]{2}{*}{ Sgr B2(N) $\mathrm{H}_{2} \mathrm{O}$ Proper Motions } & $\underline{7.1}$ & \pm & 1.1 \\
\hline & 7.1 & \pm & 1.1 \\
\hline \multicolumn{4}{|l|}{ Centroid of Distributions: } \\
\hline Globular Clusters & 8.2 & \pm & 1.1 \\
\hline RR LyraeVariables & 7.7 & \pm & 0.7 \\
\hline \multirow[t]{2}{*}{ Red Giants } & $\underline{8.6}$ & \pm & 1.7 \\
\hline & 7.9 & \pm & 0.6 \\
\hline \multicolumn{4}{|l|}{ Galaxy Models: } \\
\hline Nearby Stars & 8.9 & \pm & 0.5 \\
\hline OB Stars & 9.1 & \pm & 1.0 \\
\hline Cepheids & 7.8 & \pm & 0.7 \\
\hline HI \& HII Regions & 8.1 & \pm & 0.6 \\
\hline OH/IR Stars & 8.1 & \pm & 1.1 \\
\hline Sgr A* Proper Motions & 8.5 & \pm & 1.0 \\
\hline \multirow[t]{2}{*}{ Disk Modelling } & $\underline{8.1}$ & \pm & 1.5 \\
\hline & 8.4 & \pm & 0.3 \\
\hline \multicolumn{4}{|l|}{ Eddington Luminosity: } \\
\hline X-ray Bursters & 6.0 & \pm & 1.0 \\
\hline \multirow[t]{2}{*}{ Cyg X3 } & $\underline{7.7}$ & \pm & 1.0 \\
\hline & & \pm & \\
\hline
\end{tabular}

Table 6 summarizes the results presented in Table 5 by group. In addition to the formal statistical error $\left(\sigma_{\text {stat }}\right)$, Table 6 contains an estimate of the systematic error $\left(\sigma_{s y s}\right)$ likely for the group value. For example, the $1.0 \mathrm{kpc}$ systematic error associated with the $R_{o}$ estimate from the Centroid of Distributions method (applied to globular clusters, RR Lyrae variables, and red giants) is primarily due to an uncertainty of $\approx 0.3$ in the absolute magnitudes of RR Lyrae variables. We combine the statistical and systematic errors in quadrature and calculate a weighted average 
of the values of $R_{o}$ for the four groups. This approach yields

$$
R_{o}=7.7 \pm 0.7 \mathrm{kpc},
$$

(This value for $R_{o}$ does not contain any estimate of possible bias from a "bandwagon effect" mentioned above.)

Table 6. "Best Value" for $R_{o}$

$\begin{array}{ll}\text { METHOD } & \begin{array}{l}R_{o} \pm\left(\sigma_{\text {stat }}^{2}+\sigma_{\text {sys }}^{2}\right)^{\frac{1}{2}} \\ (\mathrm{kpc})\end{array} \\ \text { Direct Measurement } & 7.1 \pm\left(1.1^{2}+1.0^{2}\right)^{\frac{1}{2}} \\ \text { Centroid of Distributions } & 7.9 \pm\left(0.6^{2}+1.0^{2}\right)^{\frac{1}{2}} \\ \text { Galaxy Models } & 8.4 \pm\left(0.3^{2}+1.2^{2}\right)^{\frac{1}{2}} \\ \text { Eddington Luminosity } & 6.9 \pm\left(0.7^{2}+2.0^{2}\right)^{\frac{1}{2}} \\ \text { WEIGHTED AVERAGE } & 7.7 \pm 0.7\end{array}$

\section{Tomorrow's $R_{o}$}

The accuracy of $R_{o}$ estimates will improve as a consequence of several current studies. Preliminary results from $\mathrm{H}_{2} \mathrm{O}$ proper motions in a second $\mathrm{Sgr} \mathrm{B} 2$ maser source and in the W49 source, which is near the solar circle, are very promising. Other work on maser sources involves extending the OH/IR stars method to objects near the Galactic Center (see Cohen et al. these proceedings).

The advent of optical telescopes in space (Hipparcos and the Hubble Space Telescope) should lead to improved distance calibrations, for example, through better proper motions and the resolution of individual stars in clusters. Finally, a true (Earth's orbit) trigonometric parallax to $\mathrm{Sgr} \mathrm{A}^{*}$ is within the reach of the new VLBI arrays being constructed in Australia and the United States. 


\section{REFERENCES}

Backer, D. C. and Sramek, R. A. 1986, in AIP Conference Proc. 155, ed. D. C. Backer, (AIP, New York), 163.

Blanco, V. M. and Blanco, B. M. 1985, Mem. S. A. It., p15.

Bolona, L. A. and Feast, M. W. 1974, Mon. Not. R. Astr. Soc., 167, 621.

Brand, J. A. 1986, Ph.D. Thesis, Leiden.

Byl, J. and Ovenden, M. W. 1978, Ap. J., 225, 496.

Caldwell, J. A. R. and Coulson, I. M. 1987, Astron. J., 93, 1090.

Caldwell, J. A. R. and Ostriker, J. P. 1981, Ap. J., 251, 61.

Clube, S. V. M. and Dawe, J. A. 1980, Mon. Not. R. Astr. Soc., 190, 591.

Crampton, D., Bernard, D., Harris, B. L. and Thackeray, A. D. 1976, Mon. Not. R. Astr. Soc., 176, 683.

Cruz-González, C. 1974, Mon. Not. R. Astr. Soc., 168, 41.

de Vaucouleurs, G. 1983, Ap. J., 268, 451.

de Vaucouleurs, G. and Buta, R. 1978, Astron. J., 83, 1383.

Dickey, J. M. 1983, Ap. J. (Letters), 273, L71.

Ebisuzaki, T., Hanawa, T. and Sugimoto, D. 1984, P.A.S. Japan, 36, 551.

Frenk, C. S. and White, S. D. M. 1982, Mon. Not. R. Astr. Soc., 198, 173.

Glass, I. S. and Feast, M. W. 1982, Mon. Not. R. Astr. Soc., 198, 199.

Harris, W. E. 1976, Astron. J., 81, 1095.

Harris, W. E. 1980, in IAU Symposium No 85: Star Clusters, ed. J. E. Hesser, (D. Reidel; Dordrecht, Holland), 81.

Herman, J., Baud, B., Habing, H. J. and Winnberg, A. 1985, Astron. Astroph., 143, 122.

Kerr, F. J. and Lynden-Bell, D. 1986, Mon. Not. R. Astr. Soc., 221, 1023.

Molnar, L. M. 1985, Ph. D. Thesis, Harvard University.

Molnar, L. M., Reid, M. J., Grindlay, J. E., and Willner, S. 1988, in preparation.

Oort, J. H. and Plaut, L. 1975, Astron. Astroph., 41, 71.

Quiroga, R. J. 1980, Astron. Astroph., 92, 186.

Reid, M. J., Schneps, M. H., Moran, J. M., Gwinn, C. R., Genzel, R., Downes, D. and Ronnang, B. 1988, Ap. J., 330, 809.

Rohlfs, K., Chini, R., Wink, J. E. and Böhme, R. 1986, Astron. Astroph., 158, 181.

Rybicki, G., Lecar, M. and Schaefer, M. 1974, Bull. Am. Astr. Soc., 6, 453.

Sasaki, T. and Ishizawa, T. 1978, Astron. Astroph., 69, 381.

Shapley, H. 1918, Ap. J., 48, 154.

Surdin, V. G. 1980, Sov. Astron., 24, 550.

Thackeray, A. D. 1972, Quart. J. Roy. Astron. Soc., 13, 243.

Toomre, A. 1972, Quart. J. Roy. Astron. Soc., 13, 241.

Trimble, V. 1986, Comments Astrophys, 11, No 5, p257.

van den Bergh, S. and Herbst, E. 1974, Astron. J., 79, 603.

Walker, A. R. and Mack, P. 1986, Mon. Not. R. Astr. Soc., 220, 69.

Wright, A. E. and Innanen, K. A. 1972, Astron. Astroph., $21,151$. 